\title{
BMJ Open Cognitive-bias modification intervention to improve physical activity in patients following a rehabilitation programme: protocol for the randomised controlled IMPACT trial
}

\author{
Boris Cheval (D) , ${ }^{1,2}$ Axel Finckh, ${ }^{3}$ Silvio Maltagliati, ${ }^{4}$ Layan Fessler (D) ${ }^{4}$ \\ Stéphane Cullati, ${ }^{5,6}$ David Sander, ${ }^{1,2}$ Malte Friese, ${ }^{7}$ Reinout W Wiers, ${ }^{8}$ \\ Matthieu P Boisgontier (1) , 9,10 Delphine S Courvoisier (D) , ${ }^{11}$ Christophe Luthy ${ }^{12}$
}

To cite: Cheval B, Finckh A, Maltagliati S, et al. Cognitive-bias modification intervention to improve physical activity in patients following a rehabilitation programme: protocol for the randomised controlled IMPACT trial. BMJ Open 2021;11:e053845. doi:10.1136/ bmjopen-2021-053845

- Prepublication history and additional supplemental material for this paper are available online. To view these files, please visit the journal online (http://dx.doi.org/10.1136/ bmjopen-2021-053845).

Received 26 May 2021 Accepted 05 September 2021

Check for updates

(C) Author(s) (or their employer(s)) 2021. Re-use permitted under CC BY. Published by BMJ.

For numbered affiliations see end of article.

Correspondence to

Dr Boris Cheval;

boris.cheval@unige.ch

\section{ABSTRACT}

Introduction Being physically active is associated with a wide range of health benefits in patients. However, many patients do not engage in the recommended levels of physical activity (PA). To date, interventions promoting PA in patients mainly rely on providing knowledge about the benefits associated with PA to develop their motivation to be active. Yet, these interventions focusing on changing patients' conscious goals have proven to be rather ineffective in changing behaviours. Recent research on automatic factors (eg, automatic approach tendencies) may provide additional targets for interventions. However, the implementation and evaluation of intervention designed to change these automatic bases of PA are rare. Consequently, little is known about whether and how interventions that target automatically activated processes towards PA can be effective in changing PA behaviours. The Improving Physical Activity (IMPACT) trial proposes to fill this knowledge gap by investigating the effect of a cognitive-bias modification intervention aiming to modify the automatic approach towards exercise-related stimuli on PA among patients.

Methods and analysis The IMPACT trial is a singlecentre, placebo (sham controlled), triple-blinded, phase 3 randomised controlled trial that will recruit 308 patients enrolled in a rehabilitation programme in the Division of General Medical Rehabilitation at the University Hospital of Geneva (Switzerland) and intends to follow up them for up to 1 year after intervention. Immediately after starting a rehabilitation programme, patients will be randomised (1:1 ratio) to receive either the cognitive-bias modification intervention consisting of a 12-session training programme performed over 3 weeks or a control condition (placebo). The cognitive-bias modification intervention aims to improve PA levels through a change in automatic approach tendencies towards PA and sedentary behaviours. The primary outcome is the sum of accelerometer-based time spent in light-intensity, moderate-intensity and vigorous-intensity PA over 1 week after the cognitive-bias modification intervention (in minutes per week). Secondary outcomes are related to changes in (1) automatic approach tendencies and self-reported motivation to be
Strengths and limitations of this study

- The randomised controlled Improving Physical Activity trial will test the effects of an intervention based on cognitive-bias modification to improve physical activity among patients following a rehabilitation programme.

- Physical activity, sedentary behaviours, physical health and mental health will be measured at multiple time points over 1 year.

- The findings from this well-powered study will provide evidence-based recommendations for clinical interventions aiming to promote physical activity among patients in rehabilitation.

- The reliance of a single-centre trial and the selection bias due to lost to follow-up and the volunteer participation are keys limitations that may reduce our ability to generalise the results to other populations.

active, (2) physical health and (3) mental health. Sedentary behaviours and self-reported PA will also be examined. The main time point of the analysis will be the week after the end of the intervention. These outcomes will also be assessed during the rehabilitation programme, as well as $1,3,6$ and 12 months after the intervention for secondary analyses.

Ethics and dissemination The study will be conducted in accordance with the Declaration of Helsinki. This trial was approved by the Ethics Committee of Geneva Canton, Switzerland (reference number: CCER2019-02257). All participants will give an informed consent to participate in the study. Results will be published in relevant scientific journals and be disseminated in international conferences. Trial registration details The clinical trial was registered at the German clinical trials register (reference number: DRKS00023617); Pre-results.

\section{INTRODUCTION}

The health benefits of physical activity (PA) are well established and extensive. PA can 
reduce rates of cardiovascular diseases, ${ }^{1}$ cancers, ${ }^{2}$ hypertension, ${ }^{3}$ diabetes, ${ }^{4}$ obesity, ${ }^{5}$ depression ${ }^{6}$ and all-cause mortality, ${ }^{7}$ even more effectively than medication. ${ }^{8} \mathrm{PA}$ is safe and beneficial for almost everyone, while the risk of harm from moderate PA is small. ${ }^{89} \mathrm{~A}$ recent systematic review and meta-analysis suggests that any PA, irrespective of the intensity, is beneficial for health. ${ }^{7}$ In patients suffering from chronic diseases, increased PA is associated with reduced hospital admissions, decrease in pain, greater quality of life and mental health, and improvement in physical function. ${ }^{810-13}$ These myriads of benefits even led the Academy of Medical Sciences to consider PA as a miracle cure. ${ }^{14}$ Nevertheless, patients, similarly to the general population, remain largely physically inactive. ${ }^{15-17}$

Healthcare professionals are uniquely placed to promote PA among patients. Today, interventions aiming to enhance PA in patients largely relies on providing rational information about the benefits associated with PA. For example, a practical guide to help clinicians discussing about PA within a consultation has been recently proposed. ${ }^{8}$ In this guide, clinicians are encouraged to rationally address patients' concerns about PA, to explain that there are more benefits to become active than to remain sedentary, to set an achievable goal, to identify barriers to be overcome, and finally, to set a plan. This type of intervention guide is grounded in the dominant social-cognitive theories, ${ }^{18}$ which contend that goals are proximal determinants of behaviours. ${ }^{19}{ }^{20}$ From these perspectives, changing patients' conscious goals should lead to substantial changes in their behaviours. ${ }^{21}{ }^{22}$ While these types interventions have proven to be effective to change PA behaviours to some extent ${ }^{23}$ meta-analyses also indicate that these approaches are more effective in changing intentions than in changing actual behaviour. ${ }^{24}$ Thus, developing additional interventions targeting alternative mechanisms is needed.

Recent research focusing on automatic mechanisms may provide additional targets for interventions. ${ }^{25-29}$ For example, studies showed that in physically active individuals stimuli associated with $\mathrm{PA}$ attract attention, ${ }^{30-33}$ trigger positive affective reactions ${ }^{34-37}$ and activate approach tendencies towards PA. ${ }^{38-41}$ These automatically activated processes are thought to facilitate the translation of conscious goals into actual PA behaviours. Importantly, these automatic reactions predict PA behaviours above and beyond self-reported measures, such as the intention to be physically active, ${ }^{39}$ and are stronger predictors of spontaneous and unplanned actions that often consist of light-intensity PAs. ${ }^{42}$ As such, from this perspective, physical inactivity is thought to also result from an imbalance between a strong motivation to be physically active, but weak automatic approach tendencies towards PA. Crucially, this imbalance between automatic and reflective processes may be particularly pronounced in patients, whose automatic reactions towards PA may be negatively biased by the fear, pain and discomfort felt during some exercises. ${ }^{43}$ Thus, in comparison with the general population, patients may demonstrate more negative automatic reactions towards $\mathrm{PA}$, including, for example, stronger negative affective reactions and weaker approach tendencies towards PA. One practical implication of these findings is that interventions designed to promote PA in patients might particularly benefit from directly targeting automatically activated processes towards PA.

What kinds of interventions can target automatically activated processes? New types of interventions have been developed to directly target these automatic reactions towards a given health behaviour. ${ }^{44}$ For example, in alcohol addiction, studies have used a cognitive-bias modification $(\mathrm{CBM})$ intervention aimed at retraining automatic approach reactions towards alcohol using a computerised task. ${ }^{46}$ In a CBM intervention, patients were repeatedly asked to push a joystick when exposed to alcohol-related pictures, simulating an avoidance movement. Specifically, in this computerised-based task, participants were asked to push or pull a joystick in response to the format of the pictures. For example, they were instructed to make a pushing movement when the picture presented on the screen was in the landscape format (ie, avoidance), and to make a pulling movement when the picture was in the portrait format (ie, approach). To ensure congruence with the participant's actions on the joystick, the picture became smaller when the participant pushed the joystick, and it became larger when the participant pulled the joystick. Participants received training in which they had to push the joystick away in response to pictures of alcohol (ie, all alcohol pictures were presented in the push format) and to pull the joystick towards them in response to non-alcohol pictures (ie, all non-alcohol pictures were presented in the pull format). Three large studies conducted in patients showed that adding a CBM intervention to a regular cognitive-behaviour treatment yielded a beneficial effect on the relapse rates 1 year after treatment discharge, with a reduction of $9 \%,{ }^{47} 13 \%,{ }^{46}$ and $12 \%,{ }^{48}$ which could be attributed to changes in approach tendencies. ${ }^{47} 49$ These interventions have also proven to be useful in impacting cigarette smoking, ${ }^{50}$ social anxiety ${ }^{51}$ or eating behaviours. ${ }^{52-54}$ Yet, it should be noted, the clinical effectiveness of CBM interventions has been criticised, ${ }^{556}$ especially for anxiety and depressionrelated outcomes. ${ }^{57-60}$

To the best of our knowledge, however, only a handful set of studies have been conducted to target automatic processes towards PA. ${ }^{61-64}$ Crucially, only one study has been conducted to examine the effect of a brief CBM intervention targeting approach-avoidance tendencies on an exercise task in a sample of healthy young adults. ${ }^{64}$ Specifically, using a manikin task, ${ }^{42}{ }^{65}$ a variant of the approach-avoidance joystick task, participants were explicitly trained to repeatedly approach a manikin towards pictures depicting PA and to avoid pictures depicting sedentary behaviours, by pressing keys on the keyboard. Results revealed that participants spent more time exercising during a laboratory exercise task of moderate intensity (ie, doing squat), in comparison with control groups either trained to approach stimuli depicting 
sedentary behaviours and avoid stimuli depicting PA (ie, reverse contingencies) or to approach and avoid stimuli depicting PA and sedentary behaviours equally often (sham controlled). These findings suggest that a single and brief CBM session targeting automatic approach tendencies towards PA and sedentary behaviours can have beneficial effect on laboratory-based PA behaviours. However, this study has at least two important limitations. First, it is unclear if and to what extent the PA behaviour performed in the laboratory extends to behaviours performed in everyday life, thereby preventing the possibility to determine whether CMB manipulations can be effective in changing daily-life behaviours. Second, the study was conducted on a sample of rather physically active college students. As such the potential beneficial effect of adding a CBM intervention to a regular treatment in patients, a population which may particularly benefit from such manipulation, remains unknown.

\section{Objectives}

In sum, while recent research highlights the importance of targeting automatically activated processes related to PA, the effectiveness of interventions designed to change these presumed automatic bases of PA behaviours has been largely overlooked. Consequently, little is known about whether and how interventions that target automatically activated processes towards PA can be effective in changing behaviours. The primary objective of the Improving Physical Activity (IMPACT) trial is to investigate the effectiveness of a CBM intervention targeting automatic approach tendencies towards exercise-related stimuli on PA patients in a rehabilitation programme. This trial will be performed using a placebo, triple-blinded, phase 3 randomised controlled trial. The secondary objectives are to evaluate the effect of this CBM intervention on changes in (1) automatic approach tendencies and self-reported motivation to be active, (2) physical health and (3) mental health. We hypothesise that the CBM intervention will be associated with higher levels of PA (preintervention vs 1-week postintervention) (H1). Moreover, we hypothesise that the CBM intervention will increase automatic approach tendencies towards PA (H2a), but will decrease automatic approach tendencies towards sedentary behaviours (H2b). Finally, we predict that the CBM intervention will improve patients' physical and mental health (H3). All these hypotheses will also be tested during the rehabilitation programme as well as 1, 3, 6 and 12 months after the intervention (secondary analyses).

\section{METHODS AND ANALYSIS \\ Study design}

The IMPACT trial is a single-centre, placebo (sham controlled), triple-blinded, phase 3 randomised controlled trial. The trial will start (First-Participant-In) January 2022 in the ward 3DK of the Division of General Medical Rehabilitation (University Hospitals of Geneva;
Box 1 Inclusion and exclusion criteria

Inclusion criteria

- Patients treated in the ward 3DK of the Division of General Medical Rehabilitation.

- Aged 18 years or older.

- Can comply with the study protocol.

- Able to provide a written consent of participation in the trial.

Exclusion criteria

- Contraindication to physical activity in the view of the health status.

Switzerland) and will finish (Last-Participant-Out) in January 2024. The ward 3DK admits and manages patients for treatments or diagnostics evaluations, especially after being in acute care for several reasons, such as serious infections, cancer, heart failure or postsurgery follow-up treatments. This ward offers multidisciplinary treatment in rehabilitation (eg, physiotherapists, occupational therapists, nutritionists) and does not focus on improving PA engagement. In other words, within the usual care, there is not any content specifically devoted to improve patients' PA level. Eligible patients will be randomly assigned to either the CBM intervention or the active control condition (placebo) in a 1:1 ratio. The current study follows the Standard Protocol Items: Recommendations for Interventional Trials statement. ${ }^{66}$

\section{Eligibility criteria}

The eligibility criteria are listed in box 1. Participants fulfilling all the inclusion criteria are eligible for the study. The presence of the exclusion criterion will lead to the exclusion of the participant.

\section{Decision to include/exclude a participant}

The decision to include/exclude a participant from this study will be jointly decided by the chief medical officer and the research assistant.

\section{Participant screening, recruitment and consent}

All patients starting rehabilitation programme in the ward 3DK of the Division of General Medical Rehabilitation, University Hospital of Geneva, Switzerland (from January 2022 to January 2024) will be approached during the first consultation with the chief medical officer and will receive an information sheet explaining the main objective of the IMPACT trial. The investigators will explain to each participant the nature of the study, its purpose, the procedures involved, the expected duration, the potential risks and benefits and any discomfort it may entail. Each participant will be informed that the participation in the study is voluntary and that he or she may withdraw from the study at any time and that withdrawal of consent will not affect his or her subsequent medical assistance and treatment. The participant will be informed that his or her medical records may be examined by authorised individuals other than their treating physician. All participants will be provided a participant information sheet 
Table 1 Overview of the baseline screening measures

\begin{tabular}{|c|c|}
\hline Measures & Assessment method \\
\hline \multicolumn{2}{|l|}{ Inclusion criteria } \\
\hline $\begin{array}{l}\text { Patients treated in ward 3DK of the Division of General } \\
\text { Medical Rehabilitation }\end{array}$ & During the first meeting with the research assistant \\
\hline \multicolumn{2}{|l|}{$\geq 18$ years of age } \\
\hline \multicolumn{2}{|l|}{ Can comply with study protocol } \\
\hline Contraindication to PA in the view of the health status & During the first meeting with the research assistant \\
\hline \multicolumn{2}{|l|}{ Additional baseline screening assessment } \\
\hline Medical evaluation (questionnaires and objective tests) & $\begin{array}{l}\text { Patients' diseases and treatment characteristics (medical } \\
\text { burden, comorbidity, body mass index, mobility test, functional } \\
\text { independence, health-related quality of life) }\end{array}$ \\
\hline Personality & Ten-Item Personality Inventory. ${ }^{82}$ \\
\hline Expectations for improvement & $\begin{array}{l}\text { A questionnaire measuring patients' thoughts about the effects of } \\
\text { the intervention (three items: 'to what extent do you think that your } \\
\text { physical activity behaviors will improve as a result of training on the } \\
\text { computerized task?'; 'to what extent do you think that your mental } \\
\text { health will improve as a result of training on the computerized } \\
\text { task?'; 'to what extent do you think that your physical health will } \\
\text { improve as a result of training on the computerized task?'). }{ }^{67}\end{array}$ \\
\hline Self-reported motivation to change & $\begin{array}{l}\text { Questionnaire measuring patients' motivation to change their } \\
\text { condition (two items: 'how motivated are you to change your } \\
\text { health condition?'; 'to what extent do you really want to change } \\
\text { your health condition?'), to avoid a new treatment (two items: 'how } \\
\text { motivated are you to avoid a new treatment because your health } \\
\text { condition?'; 'to what extent do you really want to avoid taking a } \\
\text { new medication because of your health condition?', and to engage } \\
\text { in more PA in the future(two items: 'I intend to carry out more } \\
\text { physical activity in the next future'; I am determine to carry out } \\
\text { more physical activity in the next future'). }{ }^{66}\end{array}$ \\
\hline Self-reported ability to implement daily-life PA & $\begin{array}{l}\text { Questionnaire measuring patients' self-reported ability to adopt } \\
\text { regular PA in their daily life. Self-reported function in instrumental } \\
\text { activities of daily life (ADL; seven items), in activities of daily living } \\
\text { (ADL; seven items), and in mobility (three items). }{ }^{83}\end{array}$ \\
\hline
\end{tabular}

PA, physical activity.

and a consent form describing the study and providing sufficient information for participants to make an informed decision about their participation in the study (see online supplemental material 1 the patient consent form). Participants will have time to carefully read the documents and can give their responses up to 24 hours after having received the documents. The formal consent of a participant, using the approved consent form, will be obtained before the participant is submitted to any study procedure. Participants will then complete a first questionnaire assessing the exclusion and inclusion criteria, as well as other screening measures. All the questionnaires will be assessed electronically using REDCap software. Finally, patients' expectations regarding the effects of the intervention will be assessed. ${ }^{67}$ Table 1 provides an overview of all the baseline screening measures available. The study patient flow chart is provided in figure 1 .

\section{Sample size}

For power calculation, our intervention implements a between-subject design and random-effects statistical models (ie, t-tests). The power calculation is based on the primary outcome (ie, accelerometer-based time spent in light-intensity, moderate-intensity and vigorousintensity PA over 1 week after the CBM intervention (in minutes per week)). Based on estimates of the effect size of interventions targeting automatic approach tendencies (ie, Cohen's d=0.41; eg, a difference of $\sim 30 \mathrm{~min}$ per week between the intervention and the control group for a pooled SE of $\sim 75$ min per week), ${ }^{68} 69$ a sample size 


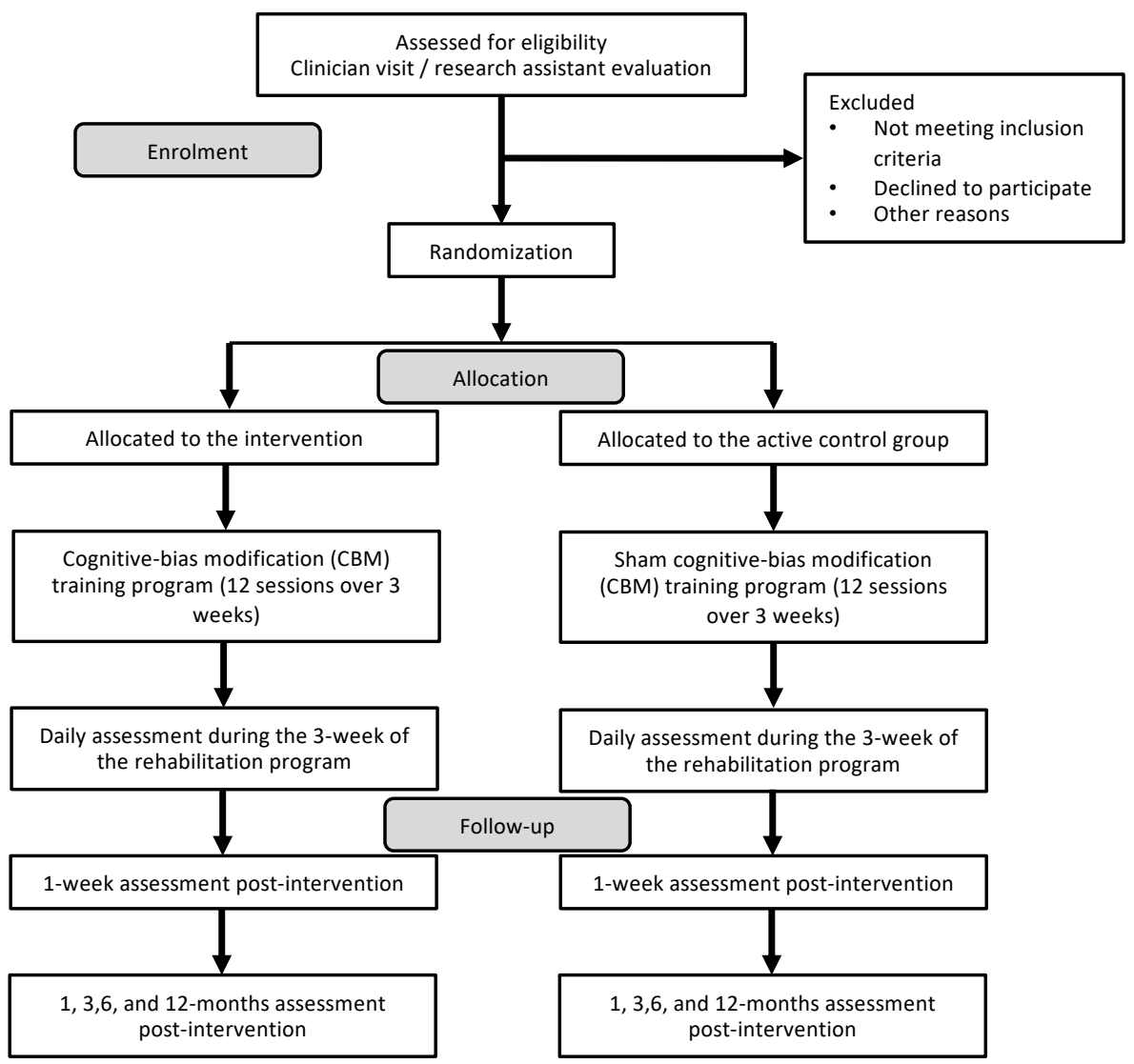

Figure 1 Flow chart note. The daily assessment refers to the measure of PA behaviours that will be continuously assessed during the rehabilitation period. The secondary outcomes will be assessed on a weekly basis. PA, physical activity.

calculation indicates that a minimum of 252 patients $(126$ per arm) would be needed to demonstrate efficacy of the intervention on the device-based PA during the week following the intervention, with a probability of committing a type I error $<5 \%$ and a probability of committing a type II error $<10 \%$. We expect a loss to follow-up of $10 \%-20 \%$ at 1 week after the intervention, and a loss of $30 \%-40 \%$ over 1 year. Thus, a minimum of 352 patients will be recruited. Of note, with this sample size, an alpha of 0.05 and a power of 0.90 , the smallest effect size we could detect is $d=0.35$. Finally, using the expected effect size (ie, $\mathrm{d}=0.41$ ), an alpha of 0.05 and a sample size of 352 patients, we obtain a power of 0.97 .

\section{Feasibility}

The ward 3DK of the Division of General Medical Rehabilitation has 24 beds and treats on average 40 patients per month. Based on the chief medical officer's experiences and a first presentation of the study to the patients treated in this unit, we expect that three patients out of five will not agree (for various reasons) to participate in the study, thereby leading to a total of about 
24 participants recruited per month. Consequently, we should be able to collect the target sample size in aapproximately 15-17 months. The average duration of participants hospitalisation in the ward 3DK is about 3 weeks. As such, though this duration can vary between patients (ie, some patients only stay a few days), this duration allows for the implementation of the whole intervention (ie, 12-session training programme performed over 3 weeks). Of note, participants who will not complete all the training sessions will still be included in the analysis. Sensibility analyses will be conducted to examine whether the number of completed sessions influence the effects of the intervention. To accelerate and facilitate knowledge dissemination, all articles will be preprinted, and data and code shared on public repositories.

\section{Patients adherence to the impact trial}

Patients adherence to the training programme (ie, if the planned training session is completed or not, and why in case of no completion) and to the other measures are documented in an electronic case report form (eCRF) powered by REDCap. ${ }^{70}$ To promote patient retention and complete follow-up (ie, 1, 3, 6 and 12 months after the end of the intervention), participants will be contacted by phone by a research assistant 2 weeks before the follow-up measurement. If they do not answer, they will receive up to two additional phone calls this week. If they do not answer, this procedure will be repeated the following week. If they still do not answer, this time of measurement will be considered as missing. Patients with missing data at a given wave will be contacted for the following waves through the above-mentioned procedure.

Patients who did not answer a given time of measurement, will still be contacted to participate in the following time points.

\section{Interventions}

All newly admitted patients will attend a meeting organised in the unit. The objective of this meeting will be to present and illustrate the health benefits of PA. Consistent with the recent practical guide to help healthcare professionals promoting PA to patients, ${ }^{8}$ research assistants will follow the 'Ask-Assess-Advise' structure for discussing PA behavioural change in the consultation. Patients will also receive a watch tracking (ie, polar) during the rehabilitation period and giving personalised feedback on their PA and sedentary behaviours. This procedure aims at increasing their self-reported motivation to be active, thereby allowing to examine the additional effects of the CBM intervention.

Intervention group: Training programme of 12 sessions over 3 weeks (ie, 4 sessions by week on average) using an adapted version of the Visual-Approach/Avoidanceby-the-Self Task, ${ }^{71}$ a task that have shown to produce large and replicable effects, compared with the manikin task. Specifically, patients will be asked to react to the format (ie, portrait vs landscape format) of the pictures depicting PA and of sedentary behaviours by pressing twice the 'move forward' or 'move backward' key press to approach or avoid the pictures, respectively. Participants will be instructed to approach the picture when it appears in a portrait format, and to avoid it when the picture appears in a landscape format (the rule will be counterbalanced between participants). Of note, unlike the previous study that relied on an explicit instruction task (ie, participants were asked to respond to the content of the pictures),${ }^{64}$ the current study uses an irrelevant feature task (ie, participants were asked to respond to the format of the pictures). This irrelevant feature task allows a training without explicit instruction. Congruent with the patient's approach or avoidance response, the whole visual environment will zoom in on the picture to simulate an approach movement and zoom out to simulate an avoidance movement. A change by $10 \%$ after each key press will be used to give the impression to walk forward or backward as a consequence of the responses. Participants in the intervention group will receive training in which $90 \%$ of pictures depicting PA will be presented in the approach format (and 10\% in the avoidance format), and $90 \%$ pictures depicting sedentary behaviours will be presented in the avoidance format (and 10\% in the approach format). This $90 / 10$ split aims to increase the patients blinding to the condition in which they will be assigned. Each training session will consist of 144 trials for a total duration of approximately $10 \mathrm{~min}$. At the first session and at the beginning of each week, the training session will be preceded by 96 assessment trials in which the contingency of approaching or avoiding PA or sedentary behaviours will be $50 \%$. Assessment trials will allow to measure patients' automatic approach-avoidance tendencies towards PA and sedentary behaviours (see figure 2).

Comparator group: Patients in the comparator group (placebo; sham controlled) will not be trained to approach PA and to avoid sedentary behaviours. Specifically, the retraining sessions will also consist of 144 trials, but the task will require an equal number of approach and avoidance responses to both stimuli depicting PA and sedentary behaviours (see figure 2). The use of a placebo was chosen to ensure that the potential effects of the experimental condition will be attributable to the content of the training programme (ie, learn to systematically approach PA-related stimuli and avoid sedentary behaviours-related stimuli) rather than because of a simple exposition effect (ie, the fact to be exposed longer to contents related to PA and sedentary behaviours).

Stimuli: Stimuli representing PA and sedentary behaviours will be created using the Unity software. A set of 195 pictures including 14 avatars ( $50 \%$ women) in either active (walking and running) and inactive posture (sit on a cubicle) will be tested in a pilot study to identify the 48 pictures the most associated with 'movement and physically active behaviours' and the 48 pictures the most associated with 'rest and physically inactive behaviours' using two Visual Analogue Scale (VAS 1; please indicate how this image is, in your opinion, associated with a behaviour that requires: $0=$ no physical exertion at all, 


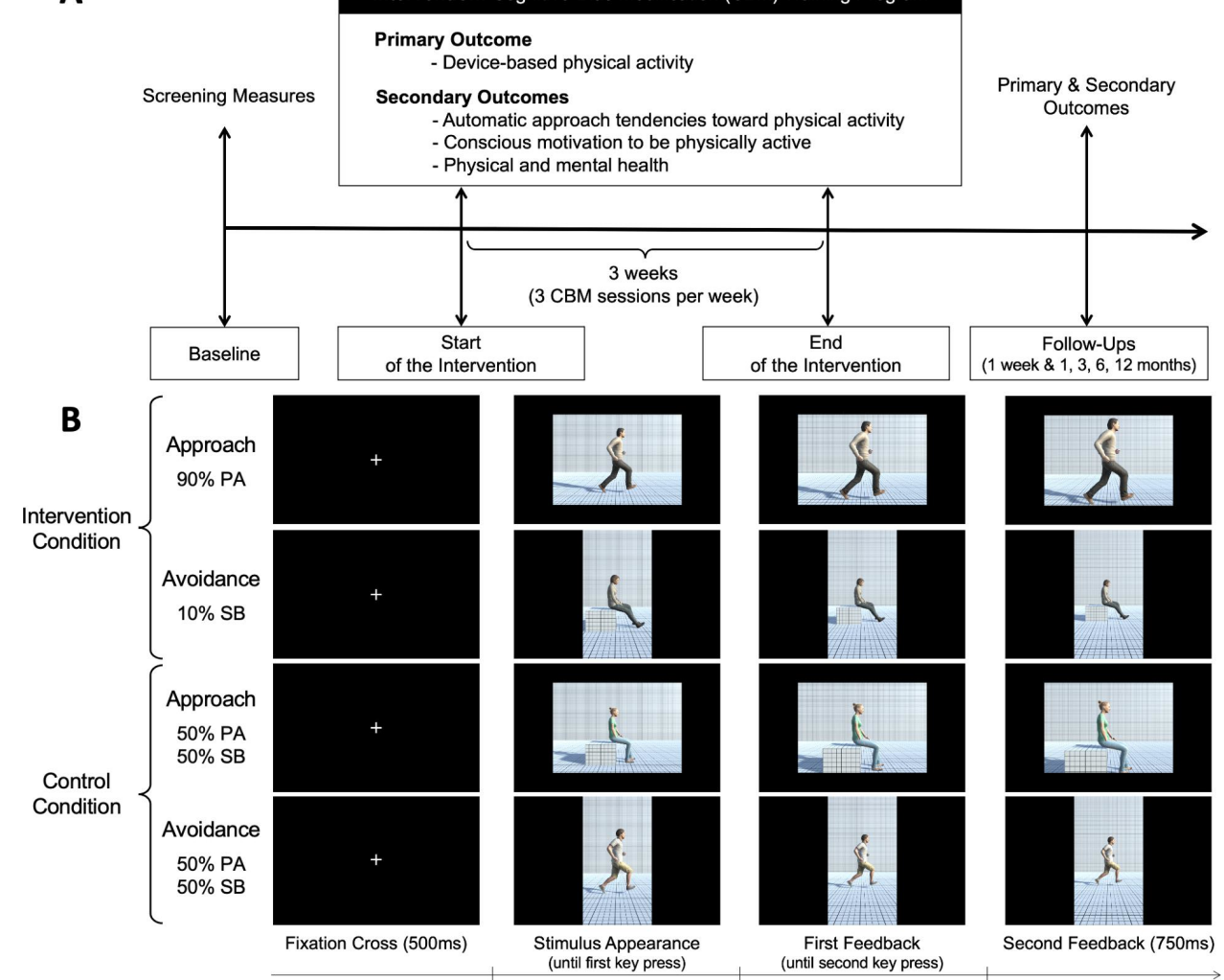

Figure 2 Study design and of cognitive-bias modification (CBM) task. Note: (A) Study design. (B). Illustration of the CBM task. In the CBM task, participants are asked to approach or avoid the picture appearing on the screen depending on its format (ie, portrait vs landscape format, counterbalanced across participants). Participants are asked to approach the picture in the approach conditions and to avoid the picture in the avoidance conditions. In the intervention condition, $90 \%$ of the pictures depicting physical activity are presented in the approach format (10\% in avoidance format), and $90 \%$ of the pictures depicting sedentary behaviours are presented in the avoidance format (10\% in approach format). In the control condition, the pictures depicting physical activity and sedentary behaviours are equally distributed across formats (ie, $50 \%-50 \%$ ). PA, physical activity.

$100=\mathrm{alot}$ of physical exertion'; VAS 2; 'please indicate how closely this image is associated with: $0=$ resting, sedentary behaviour, 100=movement, very active behaviour'). The credibility of the pictures will also be tested ('how realistic do you think this person's behavior is? Realistic meaning that the images may resemble to a real-life behavior'; on a VAS from $0=$ behaviour not at all realistic; $100=$ behaviourvery realistic) and for agreeableness ( how pleasant/sympathetic do you find the person in this image? For example, would you like to talk to her/him ; from $0=$ very unpleasant/antipathetic, $100=$ very pleasant/ sympathetic). The aim of this pilot study was twofold. First, to ensure that the selected pictures reflect the concept of interest (ie, movement and PA vs rest and physical inactivity). Second, to check that the selected pictures were equivalent in term of credibility and agreeableness across categories (ie, movement vs rest). Pictures will be built to match for colour, brightness and visual complexity. To examine the generalisation of training effects, ${ }^{72}$ in both the intervention and comparator group, only half of the pictures used in the assessment phase will be included on the training phase (the selected pictures will be counterbalanced across participants).

\section{Randomisation and blinding}

The research assistants and the participants will be blinded to the allocation of the groups. At the end of the trial, the success of the participant blinding will be examined by asking the participants to guess in what group there were, including a percentage of certainty. Moreover, the success of research assistants blinding will be examined by asking each research assistant if they were able to detect the group (comparator vs intervention) when they conducted the data collection.

The randomisation will be generated on a computer and will be performed using permuted blocks (size $=8$ ). To ensure that the research team will be blinded to the randomisation, an independent coworker will carry out the randomisation. The patient's identification number will be used to determine the sequence of randomisation. Patients will be randomised in a 1:1 ratio between the intervention and active control condition. Unblinding is not planned during the trial as we do not see any reasons that would require either the patients or the researchers to know the group in which the patients were allocated. However, if requested by the patients, unblinding is permissible at the end of the trial. 


\section{Outcomes}

\section{Primary outcome}

The primary outcome will be the sum of accelerometerbased time spent in light-intensity, moderate-intensity and vigorous-intensity PA over 1 week after the CBM intervention (in minutes per week). Following recommendations in patients, ${ }^{73}$ a three-axis accelerometer (Actigraph GT3X+; Pensacola, USA) will be used to assess PA. Patients will be given the accelerometer and related indications during the first training session. They will be asked to wear the accelerometer for the full week and to return during the next appointment. They will be instructed on how to wear the device (ie, over the right hip, affixed to an elastic belt, preferably worn under their waistbands). Currently, the waist-mounted Actigraph is the most used device to objectively measure PA. ${ }^{74}$ One-minute epochs will be used for data analyses and non-wear time will be defined as $\geq 59$ consecutive minutes of zero counts. Daily data will be included if the wear time is $\geq 10$ waking hours per day. ${ }^{75}$ Data will be included if $\geq 4$ days met the aforementioned conditions. ${ }^{76}$ The time spent in light, moderate and vigorous PA over the week will be determined through previously validated cut points, ${ }^{77}$ in bouts lasting at least $10 \mathrm{~min}$. Then, in the week following the rehabilitation period, participants will be asked to wear the accelerometer for 1 week. The sum of times spent in light-intensity, moderate-intensity and vigorous-intensity PA during this period (in minutes per week) will be used as the primary outcome. Of note, because the duration of the rehabilitation period may strongly vary between patients, it is possible that some patients will be still in the hospital after 3 weeks, while other will leave the service sooner (eg, at 2 weeks). As such, to account for this feature and to allow comparisons between patients, the accelerometer will be scheduled to start on the Monday following their discharge from the rehabilitation unit, regardless the lengths of stay in the ward. Finally, participants will be asked to wear the accelerometer for 1 week at 1, 3, 6 and 12 months postintervention.

\section{Secondary outcomes}

The secondary outcomes will be the changes in (1) automatic approach tendencies and self-reported motivation to be active, (2) physical health and (3) mental health. Sedentary behaviours and self-reported PA will also be examined. Table 2 provides an overview of all the outcomes measures and table 3 provides the schedule of assessment.

\section{Data analysis}

Primary analyses

Statistical analyses will be performed according to the intention-to-treat principle and will abide by the Consolidated Standards of Reporting Trials (CONSORT) guidelines. Analysis will be conducted in a blinded way. We will use mean, SD, median and range values to summarise the continuous data. The primary outcome (ie, the time spent in light-intensity, moderate-intensity and vigorous-intensity PA over 1 week after the CBM intervention) will be analysed using multiple linear regressions. Specifically, to test H1, we will test whether the patients' PA level during the week after the end of the intervention will be higher in the intervention group relative to the comparator group, after adjustment for covariates (ie, age, sex and indicators of the medical evaluation during the screening assessment). To test $\mathrm{H} 2 \mathrm{a}$ and $\mathrm{H} 2 \mathrm{~b}$, we will test whether patients' automatic approach tendencies towards PA will be higher and patients' automatic approach tendencies towards sedentary behaviours will be lower in the intervention group relative to the comparator group, after adjustment for covariates. Finally, to test H3, we will test whether patients' physical and mental health during the week after the end of the intervention will be higher in the intervention group relative to the comparator group, after adjustment for covariates. Moderator analyses (ie, for motivation to change, usual level of PA, personality, expectations for improvement) will be conducted.

\section{Secondary analyses}

The aforementioned models will be tested at 1, 3, 6 and 12 months after the intervention. Moreover, to examine the effect of the intervention during the rehabilitation period, mixed effects models will be used. These models account for the nested structure of the data (ie, multiple observations within a single participant), thereby providing accurate parameter estimates with acceptable type I error rates. ${ }^{78}$ Moreover, these models do not require an equal number of observations across participants, thereby allowing participants with missing observations to be included in the analyses without the need to impute those missing data. To formally examine the effect of the intervention on the evolution of PA within the rehabilitation period, models will include interaction terms between conditions (intervention group vs comparator group) and number of days within the rehabilitation programme (linear and quadratic). The number of days should be relatively equal between patients (about 21 days) but may differ to some extent (some patients can leave earlier or other later than 21 days). A statistically significant interaction will indicate that the rate of PA change throughout the rehabilitation programme would be different across the conditions. The quadratic effect of the number of days will be included to account for potential non-linear change of PA across the rehabilitation period. This will allow, for instance, to model the possibility that the effect of the intervention will take some sessions before becoming effective or that no additional effect could be hoped after a certain number of sessions. The continuous secondary outcomes will be treated in the similar way to the primary outcome. All analyses will be conducted using R software. Any deviation from the original statistical plan will be described and justified in the final trial report.

\section{Data security, management and monitoring}

Project data will be handled with uttermost discretion and will be only accessible to authorised personnel who 


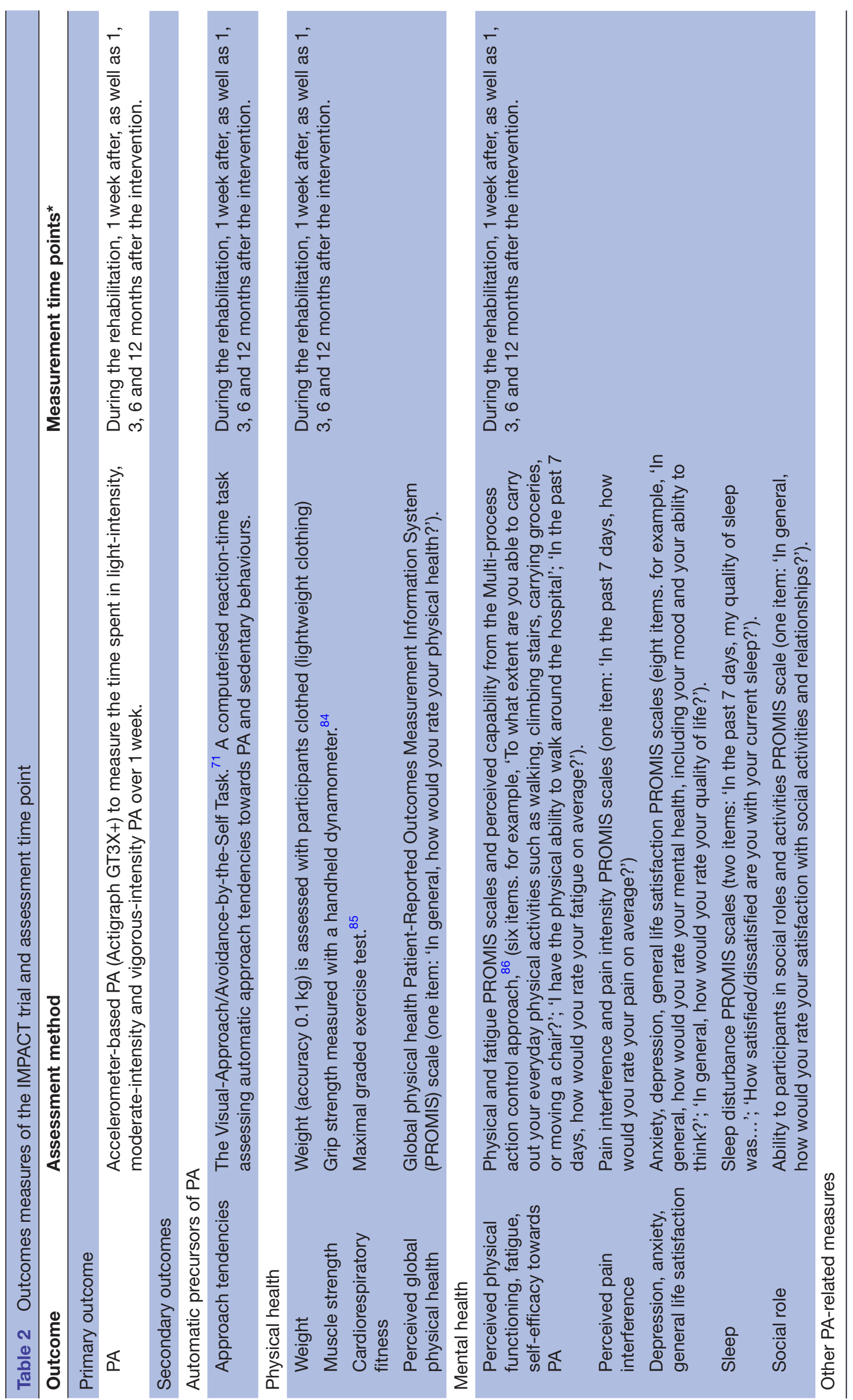




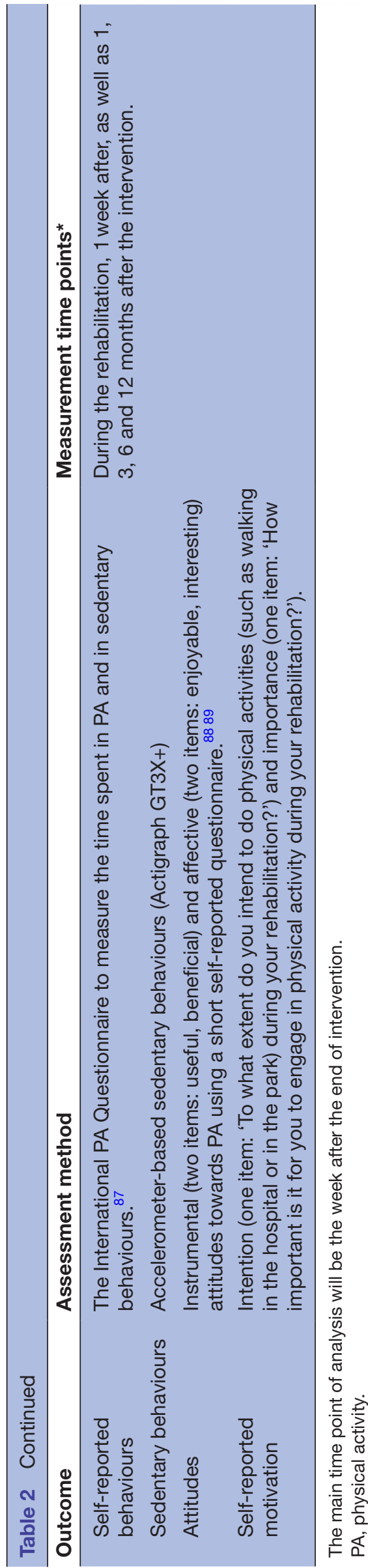

require the data to fulfil their duties within the scope of the research project. On the online CRFs and other specific documents, participants are only identified by a unique participant number. The online CRF will be created using REDCap.

Data recording: The dataset will be accompanied by a README file, which will describe the directory hierarchy and file naming convention. The directory will contain an INFO file describing the experimental protocol used in that experiment. This INFO file will also record any deviation from the protocol and other useful contextual information. This procedure should allow the data to be easily understood by other researchers and should support future reuse of the data. Metadata will be created to provide contextual information required to interpret data. This metadata file will be created in accordance with the data documentation initiative. In particular, the metadata file will include short unique identifier, the name of the author(s), the content, the date of creation, the locations, the reason why the data were generated, and how the data were created. The codebook will explicitly indicate the name, explanations and the modalities of the different variables measured in the experiment. In addition, it will include information on the study design and contain all information necessary for another analyst to use the data accurately.

Data anonymisation: Individual participant information collected during the study is considered confidential and disclosure to third parties is prohibited. Subject confidentiality will be ensured by using subject identification code numbers to correspond to treatment data in the computer files. Only a minority of personnel (ie, the principal investigator and chief medical officer) will have access to the data in a non-coded form.

Data storage: Participant data on a secure database in accordance with the General Data Protection Regulations (2018). Three copies of the data will be stored. First, original data will be stored on the principal investigator's computer, which will be backed up daily, and protected by a password. Additionally, data will be stored on a secure server hosted by the University of Geneva. Finally, data will be stored on an external device at a different location and be protected by a password. The original notebook will be stored in the principal investigator's laboratory. Local version of the data for statistical analysis will remain on a university computer, and be password protected. Each person who collected the data will have the responsibility to annotate their data within the metadata. Nevertheless, the principal investigator will have the responsibility to weekly check that the data is properly processed, documented and stored. All study data will be archived for a minimum of 10 years beyond the end of the randomised controlled trial.

Trial monitoring: The PI will organise a proper training of all involved study personnel to ensure that the study will be conducted according to the protocol. Research assistants should understand the detailed contents of the protocol before starting the data collection. For quality assurance the ethics committee may visit the research sites. Direct access to the source data and all project-related 


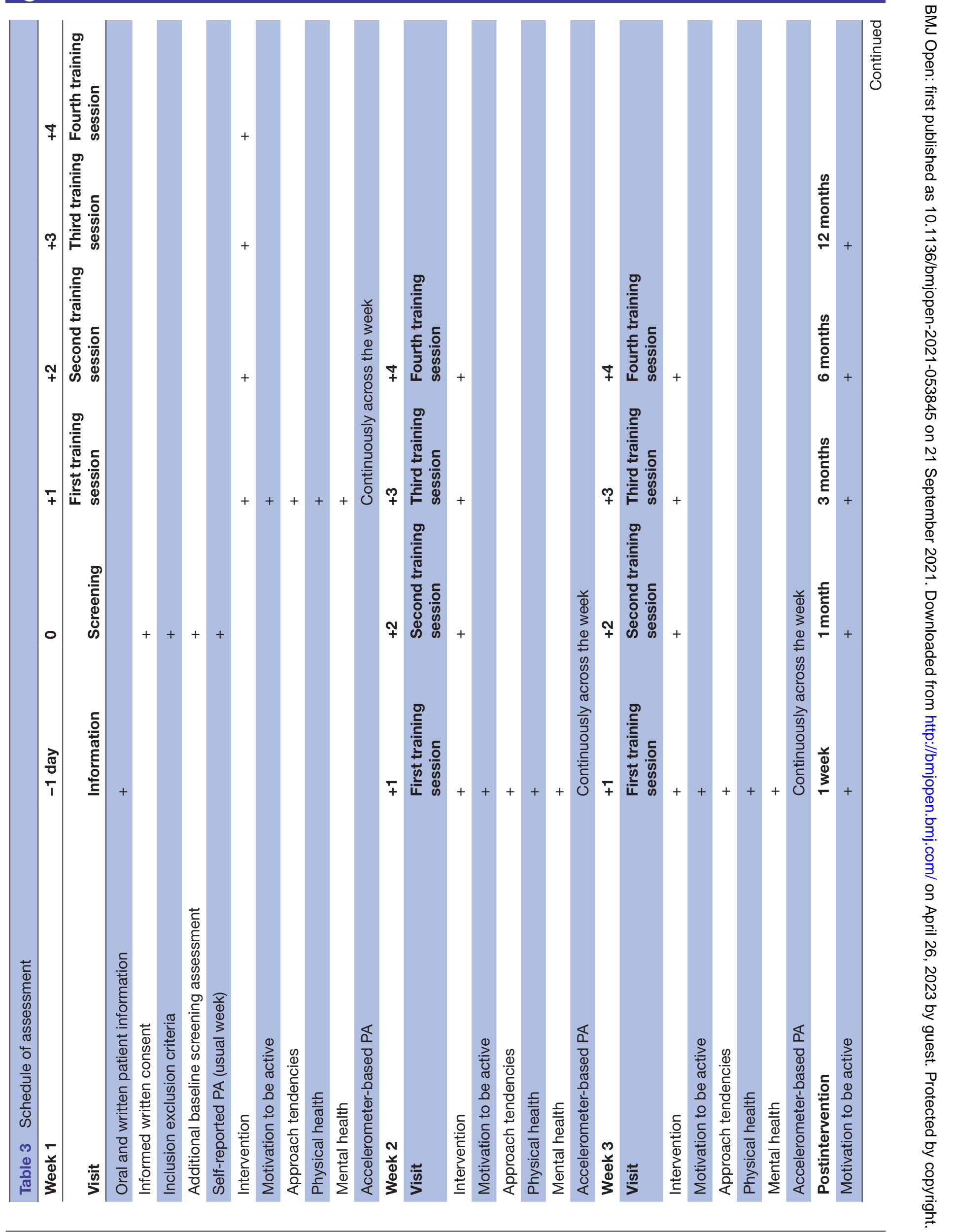




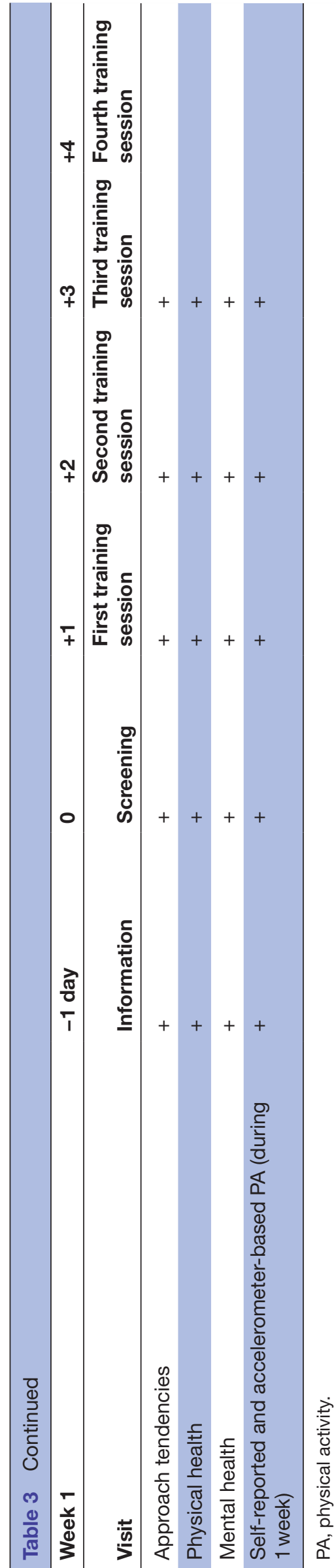

files and documents must be granted on such occasions. The principal investigator or any other competent authority may terminate the study prematurely according to the following circumstances: ethical concerns, insufficient participants recruitment, early evidence of harm or benefit of the experimental intervention through the interim analysis planned at 6 months after the start of the trial. Although no serious adverse event resulting from the intervention is expected, all potential adverse events will be documented within the eCRF.

\section{Patient and public involvement in the trial design}

No patient or public was involved in the present study.

\section{Ethics and dissemination}

The study was approved by the Ethics Committee of Geneva Canton, Switzerland (reference number: CCER2019-02257). All participants will give an informed consent to participate in the study.

Results will be published in relevant scientific journals and be disseminated in international conferences. Anonymity of the participants will be guaranteed when presenting the data at scientific meetings or publishing them in scientific journals. Individual participant information collected during the study is considered confidential and disclosure to third parties is prohibited.

Data sharing and reuse: Datasets and metadata from this trial will be deposited in ZENODO (a generic and free repository based at CERN, Geneva), and made public at the time of publication. Data in the repository will be stored in accordance with funder and university data policies. Particularly, original datasets, original software script and code, and original raw data will be deposited. However, as stressed above, personal data will be anonymised before diffusion.

\section{DISCUSSION}

PA is associated with a wide range of health benefits, ${ }^{1-7}$ but patients, similarly to the general population, remain largely physically inactive. Promoting PA to patients is thus urgently warranted, and healthcare professionals are uniquely placed to do so. ${ }^{8}$ To date, interventions mainly rely on providing rational information to change patients' conscious goals and motivation to be active. Yet, these approaches are insufficient to substantially impact actual behaviours. ${ }^{24}$ One explanation for this lack of effectiveness draws on recent observations suggesting that automatic reactions towards exercise-related stimuli are involved in the regulation of PA. ${ }^{33} 34397980$ As such, developing interventions targeting both reflective (eg, motivation) and automatic (eg, approach tendencies) precursors of PA could be particularly effective. This protocol paper outlines the design of the IMPACT trial, the first placebo, triple-blinded, randomised controlled trial examining the effectiveness of a CBM intervention targeting automatic approach tendencies towards exercise-related stimuli on PA in patients in rehabilitation 
programme remains. The IMPACT trial will focus on an accelerometer-based measure of PA as the primary outcome due to all the extensive benefits associated with being physically active. The secondary outcomes will allow examining other positive-side effects of the intervention on physical and mental health.

The IMPACT randomised controlled trial has several strengths. First, it is the first randomised controlled trial investigating the beneficial effect of an easy deliverable CBM intervention promoting PA among patients enrolled in a multidisciplinary rehabilitation programme. Second, this CBM intervention is anchored within the dual-process models of behaviour, arguing that automatic reactions towards PA represent additional targets for interventions. Accordingly, this trial will examine for the first time the efficacy of these new types of interventions, which directly targets the automatic precursors of PA behaviour. Third, we relied on an accelerometer-based measure of PA, which guarantee the validity and reliability of our primary outcome. Finally, in addition to PA behaviour, we will collect data on physical and mental health at multiple time points over 1 year. However, potential limitations should be noted. The first limitation is related to the fact that the trial is based on a single centre, which will limit the generalisation of the results to other centres. Second, because of the longitudinal design (ie, the main time point for the main analysis is assessed 4 weeks after the start of the intervention and additional time point for secondary analyses are assessed 1, 3, 6 and 12 months after the start of the intervention), we cannot exclude a selection bias due to attrition. Likewise, as participation in our study is voluntary, it may favour the selection of patients with a higher health status or the most motivated to engage in PA. These features are key limitations that may reduce our ability to generalise the results to other populations. Third, to reduce patients' burden, the measure of physical and mental health is based on a single or few items, which may reduce the reliability and validity of these secondary outcomes' measurement. Finally, the rehabilitation programme in the Division of General Medical Rehabilitation is a programme receiving patients that have been in acute care for different reasons such as serious infections, cancer, heart or lung failure or postsurgery follow-up treatments. Accordingly, the profiles of the patients included in the trial may strongly differ from one patient to another. Therefore, although patients' profile (eg, age, sex or features of the medical evaluation) will be adjusted in the model, the diversity of those profiles may still produce a level of variability likely to influence the effects of the intervention.

$\mathrm{PA}$ is a key factor to improve the management of patients' diseases. Helping patients to become more active is likely to promote their recovery, their physical and mental health, as well as to reduce the development of other comorbidities. Targeting automatic reactions towards PA, which may be negatively biased in patients, is particularly innovative. Furthermore, this low cost and easily deliverable intervention could be rapidly implemented on a large scale to help patients become more physically active. The findings from this study will provide evidence-based conclusions for future interventions promoting $\mathrm{PA}$ in patients.

\section{Author affiliations}

${ }^{1}$ Swiss Center for Affective Sciences, University of Geneva, Geneva, Switzerland

${ }^{2}$ Laboratory for the Study of Emotion Elicitation and Expression (E3Lab), Department of Psychology, University of Geneva, Geneva, Switzerland

${ }^{3}$ Division of Rheumatology, Department of Medicine, Geneva University Hospitals, Geneva, Switzerland

${ }^{4}$ Laboratory SENS, Department of Sport Science, University Grenoble Alpes, Saint-

Martin-d'Heres, France

${ }^{5}$ Population Health Laboratory, Department of Medicine, University of Fribourg, Fribourg, Switzerland

${ }^{6}$ Department of Readaptation and Geriatrics, University of Geneva, Geneva, Switzerland

${ }^{7}$ Department of Psychology, Saarland University, Saarbrucken, Germany ${ }^{8}$ Department of Psychology and center for Urban Mental Health, University of Amsterdam, Amsterdam, The Netherlands

${ }^{9}$ School of Rehabilitation Sciences, Faculty of Health Sciences, University of Ottawa, Ottawa, Ontario, Canada

${ }^{10}$ Bruyère Research Institute, Ottawa, Ontario, Canada

${ }^{11}$ Quality of Care Unit, Geneva University Hospitals, Geneva, Switzerland

${ }^{12}$ Division of General Medical Rehabilitation, University Hospitals of Geneva, Geneva, Switzerland

\section{Twitter Layan Fessler @LayanFessler}

Contributors BC: conceptualisation, writing-original draft; AF: conceptualisation, writing-review and editing; SM: Methodology-creation of the tasks, writing-review and editing; LF: writing-review and editing; SC: writing-review and editing; DS: writing-review and editing; MF: writing-review and editing; RWW: writing-review and editing; MPB: conceptualisation, writing-original draft; DSC: supervision, resources-provision on instrumentation, writing-review and editing; $\mathrm{CL}$ : supervision, resources-provision of study materials, writing-original draft.

Funding This work was supported by the Swiss National Science Foundation, grant number PZ00P1_180040. The purchase of protocol-related material (ie, 30 Polar watches and accelerometers) is provided by the non-operating fund of the Division of General Medical Rehabilitation, University Hospital of Geneva, Switzerland. The computer equipment (3 laptops) will be made available by Division of General Medical Rehabilitation, University Hospital of Geneva. MPB is supported by the Natural Sciences and Engineering Research Council of Canada (RGPIN-202103153) and by a grant from The Banting Research Foundation.

Competing interests None declared.

Patient consent for publication Consent obtained directly from patient(s)

Provenance and peer review Not commissioned; externally peer reviewed.

Supplemental material This content has been supplied by the author(s). It has not been vetted by BMJ Publishing Group Limited (BMJ) and may not have been peer-reviewed. Any opinions or recommendations discussed are solely those of the author(s) and are not endorsed by BMJ. BMJ disclaims all liability and responsibility arising from any reliance placed on the content. Where the content includes any translated material, BMJ does not warrant the accuracy and reliability of the translations (including but not limited to local regulations, clinical guidelines, terminology, drug names and drug dosages), and is not responsible for any error and/or omissions arising from translation and adaptation or otherwise.

Open access This is an open access article distributed in accordance with the Creative Commons Attribution 4.0 Unported (CC BY 4.0) license, which permits others to copy, redistribute, remix, transform and build upon this work for any purpose, provided the original work is properly cited, a link to the licence is given, and indication of whether changes were made. See: https://creativecommons.org/ licenses/by/4.0/.

\section{ORCID iDs}

Boris Cheval http://orcid.org/0000-0002-6236-4673

Layan Fessler http://orcid.org/0000-0002-8435-5110

Matthieu P Boisgontier http://orcid.org/0000-0001-9376-3071

Delphine S Courvoisier http://orcid.org/0000-0002-1956-2607 


\section{REFERENCES}

1 Wahid A, Manek N, Nichols M, et al. Quantifying the association between physical activity and cardiovascular disease and diabetes: a systematic review and meta-analysis. J Am Heart Assoc 2016;5:e002495.

2 Moore SC, Lee I-M, Weiderpass E, et al. Association of leisure-time physical activity with risk of 26 types of cancer in 1.44 million adults. JAMA Intern Med 2016;176:816-25.

3 Liu X, Zhang D, Liu Y, et al. Dose-response association between physical activity and incident hypertension: a systematic review and meta-analysis of cohort studies. Hypertension 2017;69:813-20.

4 Aune D, Norat T, Leitzmann M, et al. Physical activity and the risk of type 2 diabetes: a systematic review and dose-response metaanalysis. Eur J Epidemiol 2015;30:529-42.

5 Bleich SN, Vercammen KA, Zatz LY, et al. Interventions to prevent global childhood overweight and obesity: a systematic review. Lancet Diabetes Endocrinol 2018;6:332-46.

6 Schuch F, Vancampfort D, Firth J, et al. Physical activity and sedentary behavior in people with major depressive disorder: a systematic review and meta-analysis. J Affect Disord 2017;210:139-50.

7 Ekelund U, Tarp J, Steene-Johannessen J, et al. Dose-response associations between accelerometry measured physical activity and sedentary time and all cause mortality: systematic review and harmonised meta-analysis. BMJ 2019;366:14570.

8 Haseler C, Crooke R, Haseler T. Promoting physical activity to patients. BMJ 2019;366:I5230.

9 Godlee F. The miracle cure. BMJ 2019;366:15605.

10 Anderson L, Oldridge N, Thompson DR, et al. Exercise-based cardiac rehabilitation for coronary heart disease: cochrane systematic review and meta-analysis. J Am Coll Cardiol 2016;67:1-12.

11 Stevinson C, Lawlor DA, Fox KR. Exercise interventions for cancer patients: systematic review of controlled trials. Cancer Causes Control 2004;15:1035-56.

12 Taylor RS, Brown A, Ebrahim S, et al. Exercise-based rehabilitation for patients with coronary heart disease: systematic review and meta-analysis of randomized controlled trials. Am J Med 2004;116:682-92.

13 Zanuso S, Jimenez A, Pugliese G, et al. Exercise for the management of type 2 diabetes: a review of the evidence. Acta Diabetol 2010;47:15-22.

14 Acadamy of Medical Royal Colleges. Exercise: the miracle cure and the role of the doctor in promoting it, 2015. Available: https://www. aomrc.org.uk/wp-content/uploads/2016/05/Exercise_the_Miracle_ Cure 0215.pdf

15 Johansen KL, Chertow GM, Ng AV, et al. Physical activity levels in patients on hemodialysis and healthy sedentary controls. Kidney Int 2000;57:2564-70.

16 Watz H, Waschki B, Meyer T, et al. Physical activity in patients with COPD. Eur Respir J 2009;33:262-72.

17 Jeong S-W, Kim S-H, Kang S-H, et al. Mortality reduction with physical activity in patients with and without cardiovascular disease. Eur Heart J 2019;40:3547-55.

18 Rhodes RE, Nigg CR. Advancing physical activity theory: a review and future directions. Exerc Sport Sci Rev 2011;39:113-9.

19 Bandura A. Self-efficacy: the exercise of control (1st edition). New York: Worth Publishers, 1997.

20 Carver CS, Scheier MF. On the self-regulation of behavior. New York: Cambridge University Press, 1998.

21 Sheeran P, Gollwitzer PM, Bargh JA. Nonconscious processes and health. Health Psychol 2013;32:460-73.

22 Brand R, Cheval B. Theories to explain exercise motivation and physical inactivity: ways of expanding our current theoretical perspective. Front Psychol 2019;10:1147.

23 Hillsdon M, Foster C, Thorogood M. Interventions for promoting physical activity. Cochrane Database Syst Rev 2005;25:CD003180.

24 Rhodes RE, Dickau L. Experimental evidence for the intentionbehavior relationship in the physical activity domain: a meta-analysis. Health Psychol 2012;31:724-7.

25 Cheval B, Radel R, Neva JL, et al. Behavioral and neural evidence of the rewarding value of exercise behaviors: a systematic review. Sports Med 2018;48:1389-404.

26 Brand R, Ekkekakis P. Affective-reflective theory of physical inactivity and exercise. Ger J Exerc Sport Res 2018;48:48-58.

27 Conroy DE, Berry TR. Automatic affective evaluations of physical activity. Exerc Sport Sci Rev 2017;45:230-7.

28 Cheval B, Sarrazin P, Boisgontier MP. Temptations toward behaviors minimizing energetic costs (BMEC) automatically activate physical activity goals in successful exercisers. Psychol Sport Exerc 2017;30:110-7.
29 Cheval B, Boisgontier MP. The theory of effort minimization in physical activity. Exerc Sport Sci Rev 2021;49:168-78.

30 Berry TR. Who's even interested in the exercise message? Attentional bias for exercise and sedentary-lifestyle related words. $J$ Sport Exerc Psychol 2006;28:4-17.

31 Berry TR, Spence JC, Stolp SM. Attentional bias for exercise-related images. Res Q Exerc Sport 2011;82:302-9.

32 Calitri R, Lowe R, Eves FF, et al. Associations between visual attention, implicit and explicit attitude and behaviour for physical activity. Psychol Health 2009;24:1105-23.

33 Cheval B, Miller MW, Orsholits D, et al. Physically active individuals look for more: an eye-tracking study of attentional bias. Psychophysiology 2020;57:e13582.

34 Conroy DE, Hyde AL, Doerksen SE, et al. Implicit attitudes and explicit motivation prospectively predict physical activity. Ann Behav Med 2010;39:112-8.

35 Rebar AL, Ram N, Conroy DE. Using the EZ-diffusion model to score a single-category implicit association test of physical activity. Psychol Sport Exerc 2015;16:96-105.

36 Bluemke M, Brand R, Schweizer G, et al. Exercise might be good for me, but I don't feel good about it: do automatic associations predict exercise behavior? J Sport Exerc Psychol 2010;32:137-53.

37 Chevance G, Caudroit J, Romain AJ, et al. The adoption of physical activity and eating behaviors among persons with obesity and in the general population: the role of implicit attitudes within the theory of planned behavior. Psychol Health Med 2017;22:319-24.

38 Hannan TE, Moffitt RL, Neumann DL. Implicit approach-avoidance associations predict leisure-time exercise independently of explicit exercise motivation. Sport Exerc Perform Psychol 2019;8:210-22.

39 Cheval B, Sarrazin P, Isoard-Gautheur S, et al. Reflective and impulsive processes explain (in)effectiveness of messages promoting physical activity: a randomized controlled trial. Health Psychol 2015;34:10-19.

40 Cheval B, Sarrazin P, Isoard-Gautheur S. How impulsivity shapes the interplay of impulsive and reflective processes involved in objective physical activity. Pers Individ Dif 2016;96:132-7.

41 Cheval B, Tipura E, Burra N, et al. Avoiding sedentary behaviors requires more cortical resources than avoiding physical activity: an EEG study. Neuropsychologia 2018;119:68-80.

42 Cheval B, Sarrazin P, Pelletier L. Impulsive approach tendencies towards physical activity and sedentary behaviors, but not reflective intentions, prospectively predict non-exercise activity thermogenesis. PLoS One 2014;9:e115238.

43 Shelby RA, Somers TJ, Keefe FJ, et al. Domain specific self-efficacy mediates the impact of pain catastrophizing on pain and disability in overweight and obese osteoarthritis patients. J Pain 2008;9:912-9.

44 Friese M, Hofmann W, Wiers RW. On taming horses and strengthening riders: recent developments in research on interventions to improve self-control in health behaviors. Self Identity 2011;10:336-51.

45 Marteau TM, Hollands GJ, Fletcher PC. Changing human behavior to prevent disease: the importance of targeting automatic processes. Science 2012;337:1492-5.

46 Wiers RW, Eberl C, Rinck M, et al. Retraining automatic action tendencies changes alcoholic patients' approach bias for alcohol and improves treatment outcome. Psychol Sci 2011;22:490-7.

47 Eberl C, Wiers RW, Pawelczack S, et al. Approach bias modification in alcohol dependence: do clinical effects replicate and for whom does it work best? Dev Cogn Neurosci 2013;4:38-51.

48 Manning V, Garfield JBB, Staiger PK, et al. Effect of cognitive bias modification on early relapse among adults undergoing inpatient alcohol withdrawal treatment: a randomized clinical trial. JAMA Psychiatry 2021;78:133-40.

49 Gladwin TE, Rinck M, Eberl C, et al. Mediation of cognitive bias modification for alcohol addiction via stimulus-specific alcohol avoidance association. Alcohol Clin Exp Res 2015;39:101-7.

50 Wittekind CE, Feist A, Schneider BC, et al. The approach-avoidance task as an online intervention in cigarette smoking: a pilot study. $J$ Behav Ther Exp Psychiatry 2015;46:115-20.

51 Taylor CT, Amir N. Modifying automatic approach action tendencies in individuals with elevated social anxiety symptoms. Behav Res Ther 2012;50:529-36.

52 Fishbach A, Friedman RS, Kruglanski AW. Leading US not unto temptation: momentary allurements elicit overriding goal activation. $J$ Pers Soc Psychol 2003;84:296-309.

53 Fishbach A, Shah JY. Self-Control in action: implicit dispositions toward goals and away from temptations. J Pers Soc Psychol 2006;90:820.

54 Aulbach MB, Knittle K, Haukkala A. Implicit process interventions in eating behaviour: a meta-analysis examining mediators and moderators. Health Psychol Rev 2019;13:179-208. 
55 Field M, Duka T, Eastwood B, et al. Experimental manipulation of attentional biases in heavy drinkers: do the effects generalise? Psychopharmacology 2007;192:593-608.

56 Schoenmakers T, Wiers RW, Jones BT, et al. Attentional re-training decreases attentional bias in heavy drinkers without generalization. Addiction 2007;102:399-405.

57 Cristea IA, Kok RN, Cuijpers P. The effectiveness of cognitive bias modification interventions for substance addictions: a meta-analysis. PLoS One 2016;11:e0162226.

58 Cristea IA, Kok RN, Cuijpers P. Efficacy of cognitive bias modification interventions in anxiety and depression: meta-analysis. $\mathrm{Br} \mathrm{J}$ Psychiatry 2015;206:7-16.

59 Mogoașe C, David D, Koster EHW. Clinical efficacy of attentional bias modification procedures: an updated meta-analysis. J Clin Psychol 2014;70:1133-57.

60 Boffo M, Zerhouni O, Gronau QF, et al. Cognitive bias modification for behavior change in alcohol and smoking addiction: Bayesian meta-analysis of individual participant data. Neuropsychol Rev 2019;29:52-78.

61 Markland D, Hall CR, Duncan LR. The effects of an imagery intervention on implicit and explicit exercise attitudes. Psychol Sport Exerc 2015;17:24-31.

62 Antoniewicz F, Brand R. Learning to like exercising: evaluative conditioning changes automatic evaluations of exercising and influences subsequent exercising behavior. J Sport Exerc Psychol 2016;38:138-48.

63 Qiu Y, Zhang G. Make exercise easier: a brief intervention to influence implicit attitudes towards exercise and physical activity behavior. Learning and Motivation 2020;72:101660.

64 Cheval B, Sarrazin P, Pelletier L, et al. Effect of retraining approachavoidance tendencies on an exercise task: a randomized controlled trial. J Phys Act Health 2016;13:1396-403.

65 Krieglmeyer R, Deutsch R. Comparing measures of approachavoidance behaviour: the manikin task vs. two versions of the joystick task. Cogn Emot 2010;24:810-28.

66 Chan A-W, Tetzlaff JM, Altman DG, et al. Spirit 2013 statement: defining standard protocol items for clinical trials. Ann Intern Med 2013;158:200-7.

67 Boot WR, Simons DJ, Stothart C, et al. The pervasive problem with placebos in psychology: why active control groups are not sufficient to rule out placebo effects. Perspect Psychol Sci 2013;8:445-54.

68 Treur J, van Dessel P, ter Haar A. Study protocol for ABC-training: a new, personalized cognitive training for smoking cessation. OSF 2021.

69 Van Dessel P, Hughes S, De Houwer J. Consequence-based approach-avoidance training: a new and improved method for changing behavior. Psychol Sci 2018;29:1899-910.

70 Harris PA, Taylor R, Thielke R, et al. A metadata-driven methodology and workflow process for providing translational research informatics support. J Biomed Inform 2009;42:377-81.

71 Rougier M, Muller D, Ric F. A new look at sensorimotor aspects in approach/avoidance tendencies: the role of visual whole-body movement information. J Exp Soc Psychol 2018;76:42-53.
72 Wiers RW, Rinck M, Kordts R, et al. Retraining automatic actiontendencies to approach alcohol in hazardous drinkers. Addiction 2010;105:279-87.

73 Mantri S, Wood S, Duda JE, et al. Comparing self-reported and objective monitoring of physical activity in Parkinson disease. Parkinsonism Relat Disord 2019;67:56-9.

74 Bassett DR, Troiano RP, McClain JJ, et al. Accelerometer-based physical activity: total volume per day and standardized measures. Med Sci Sports Exerc 2015;47:833-8.

75 Evenson KR, Terry JW. Assessment of differing definitions of accelerometer nonwear time. Res Q Exerc Sport 2009;80:355-62.

76 Matthews CE, Ainsworth BE, Thompson RW, et al. Sources of variance in daily physical activity levels as measured by an accelerometer. Med Sci Sports Exerc 2002;34:1376-81.

77 Freedson PS, Melanson E, Sirard J. Calibration of the computer science and applications, Inc. accelerometer. Med Sci Sports Exerc 1998;30:777-81.

78 Boisgontier MP, Cheval B. The ANOVA to mixed model transition Neurosci Biobehav Rev 2016;68:1004-5.

79 Cheval B, Cabral DAR, Daou M. Inhibitory control elicited by physical activity and inactivity stimuli: an EEG study. Motiv Sci 2021.

80 Cheval B, Daou M, Cabral DAR. Higher inhibitory control is required to escape the innate attraction to effort minimization. Psychol Sport Exerc 2020;51:101781.

81 Grimby G, Börjesson M, Jonsdottir I. The "Saltin-Grimby physical activity level scale" and its application to health research. Scand J Med Sci Spor 2015;25:119-25.

82 Gosling SD, Rentfrow PJ, Swann Jr WB. A very brief measure of the Big-Five personality domains. J Res Pers 2003;37:504-28.

83 Bravell ME, Zarit SH, Johansson B. Self-Reported activities of daily living and Performance-based functional ability: a study of congruence among the oldest old. Eur J Ageing 2011;8:199-209.

84 Leong DP, Teo KK, Rangarajan S, et al. Prognostic value of grip strength: findings from the prospective urban rural epidemiology (pure) study. Lancet 2015;386:266-73.

85 Mang CS, McEwen LM, Maclsaac JL, et al. Exploring genetic influences underlying acute aerobic exercise effects on motor learning. Sci Rep 2017;7:12123.

86 Rhodes RE. The evolving understanding of physical activity behavior: a multi-process action control approach. Advances in Motivation Science 2017;4:171-205.

87 Craig CL, Marshall AL, Sjöström M, et al. International physical activity questionnaire: 12 -country reliability and validity. Med Sci Sports Exerc 2003;35:1381-95

88 Lowe R, Eves F, Carroll D. The influence of affective and instrumenta beliefs on exercise intentions and behavior: a longitudinal analysis. $J$ Appl Soc Psychol 2002;32:1241-52.

89 French DP, Sutton S, Hennings SJ. The importance of affective beliefs and attitudes in the theory of planned behavior: predicting intention to increase physical activity 1. J Appl Soc Psychol 2005;35:1824-48. 